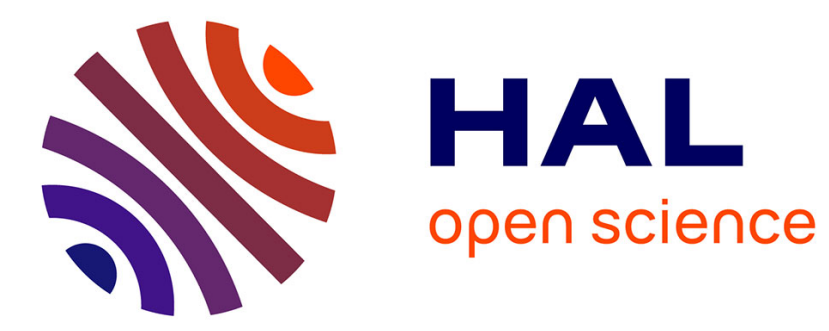

\title{
On the Eclipsing Phenomenon with Phase Codes
}

\author{
U. Tan, Olivier Rabaste, Claude Adnet, Jean-Philippe Ovarlez
}

\section{- To cite this version:}

U. Tan, Olivier Rabaste, Claude Adnet, Jean-Philippe Ovarlez. On the Eclipsing Phenomenon with Phase Codes. RADAR 2019, Sep 2019, TOULON, France. hal-02417491

\section{HAL Id: hal-02417491 \\ https://hal.science/hal-02417491}

Submitted on 18 Dec 2019

HAL is a multi-disciplinary open access archive for the deposit and dissemination of scientific research documents, whether they are published or not. The documents may come from teaching and research institutions in France or abroad, or from public or private research centers.
L'archive ouverte pluridisciplinaire HAL, est destinée au dépôt et à la diffusion de documents scientifiques de niveau recherche, publiés ou non, émanant des établissements d'enseignement et de recherche français ou étrangers, des laboratoires publics ou privés. 


\title{
On the Eclipsing Phenomenon with Phase Codes
}

\author{
U. Tan*†‡, O. Rabaste ${ }^{\ddagger}$, C. Adnet ${ }^{\dagger}$, J.-P. Ovarlez ${ }^{* \ddagger}$ \\ *Sondra, CentraleSupélec, Université Paris-Saclay, Gif-Sur-Yvette, France \\ $\dagger$ Thales Land \& Air Systems, Limours, France \\ ${ }^{\ddagger}$ DEMR, ONERA, Université Paris-Saclay, Palaiseau, France \\ e-mail: u.tan@laposte.net, olivier.rabaste@onera.fr, claude.adnet@thalesgroup.com,jean-philippe.ovarlez@onera.fr
}

\begin{abstract}
This paper is interested in phase code design, and especially in their robustness to the eclipsing phenomenon. In a radar background, the term "eclipsing" describes a partial loss of data at the reception side. It implies an increase of pulse compression sidelobes, and thus a degradation of detection performance. In this regard, this paper describes an optimisation procedure which aims to provide waveforms (phase codes) that are quite robust to the eclipsing phenomenon. Some simulations are provided to highlight the usefulness of the procedure.

Index Terms-Eclipsing, phase codes, pulse compression, waveform optimisation.
\end{abstract}

\section{INTRODUCTION}

A definition of an eclipse that can be found in a dictionary is the following [1]: "the total or partial obscuring of one celestial [sic] body by another". In a radar framework, it does not indicate that a target is hidden by another; it indicates that part of a pulse has not been recorded, as illustrated in Fig. 1.

Monostatic pulse radars use the same antenna for transmitting and receiving. Whilst one of these functions is in use, the other is turned off. It implies in particular that monostatic radars are blind during a transmission. In a time-related scale, which scenarios could be encountered ? [2]

- The ideal case consists in receiving the echo from a target before the next pulse is transmitted. Range measurement is properly performed as usual.

- If an echo from a quite long-range target arrives after the transmission of the next pulse, it might be mistakenly associated with that pulse, and not the actual one. This case is known as a range ambiguity and is depicted Fig. 1.

- Otherwise, a return may be perceived within a transmitted pulse width (Fig 1, bottom). In other words, the beginning or the end of the reflected waveform may be missed. That phenomenon is known as an "eclipse" [3]. Let us notice that only partial eclipses are considered, because a total eclipse is akin to no reception.

After all, an eclipse is defined as a partial loss of data in the reception side. This loss manifests through several forms, as depicted in Fig. 2. In this figure, matched filter outputs of a chirp and a phase code are represented, whereas the received signal has been truncated by half. As for the chirp, a Signalto-Noise Ratio (SNR) loss is observed, due to the resulting mismatched filtering (the filter applied to the received signal is no longer matched to it), as well as an enlargement of the mainlobe (due to a decrease of the remaining bandwidth). As

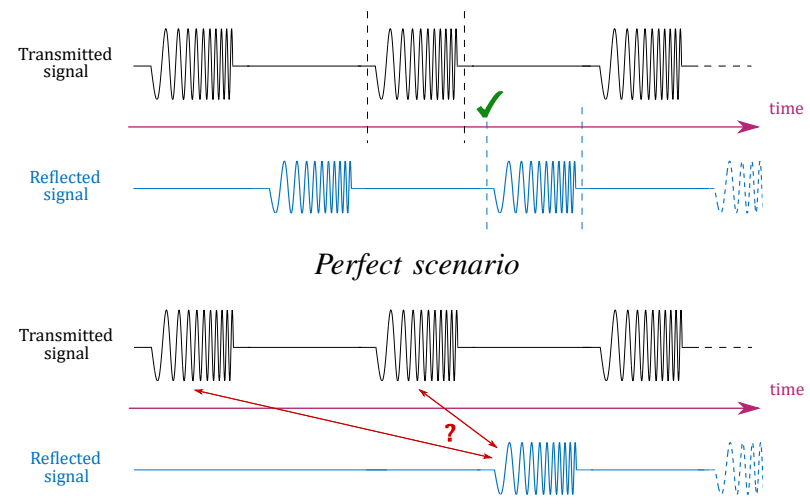

A range ambiguity

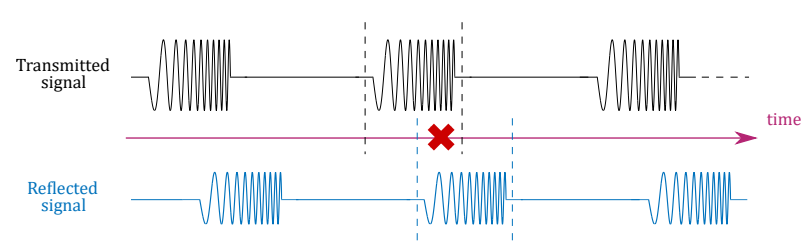

An eclipse: the echo from the target is partially received within a transmission.

Figure 1. Eclipse Phenomenon

for the polyphase code, an increase of the sidelobes is noticed (with also the same SNR loss).

This energetic loss is inherent to the fact that the processing filter is not matched to the received signal because of the eclipse (in other words, to the unsought mismatched processing). Incidentally, it is identical for each sequence of the same length under the constant modulus constraint (defined below). However, in the previous example, a linear frequency modulation preserves the sidelobe level, whilst a random phase code does not. In other words, some sequences seem to be more robust to eclipsing than others. Hence, this article is interested in designing phase codes that limit that increase of sidelobes. In order to do so, an optimisation problem is suggested: its cost function is a measure of the sidelobe level after a partial pulse compression.

This paper is organised as follows. Section II deals with eclipses at the beginning of a pulse, while Section III considers eclipses both at the beginning and at the end. Similar works have been done in [4], [3], [5], but for non-linear frequency modulations in the former. Notation: In the following, bold 

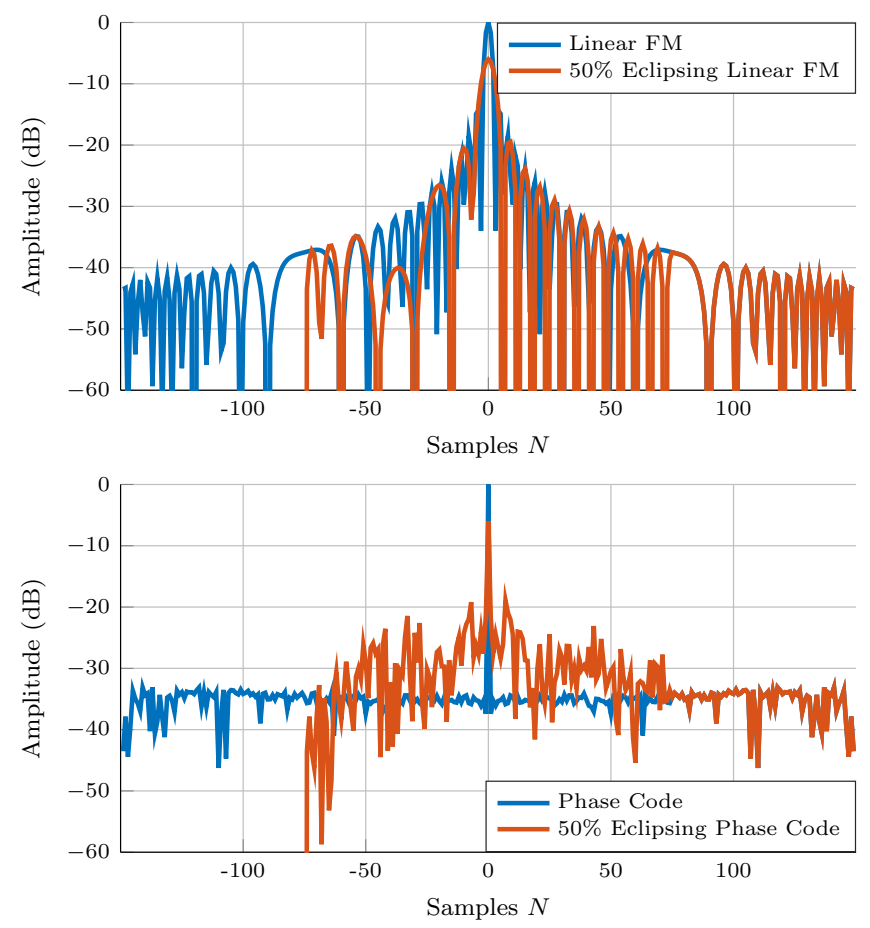

Figure 2. Correlation sequences of eclipsed signals A chirp on the top, a phase code on the bottom. Notice an enlargement of the mainlobe with the chirp, an increase of the sidelobe level and a loss because of mismatched filtering.

letters designate vectors and matrices. $(.)^{*},(.)^{T}$ and $(.)^{H}$ denote the conjugate, the transpose and the transpose conjugate operator, respectively. For two integers $a$ and $b, \llbracket \cdot, \cdot \rrbracket$ is an integer interval, that is, with $a<b, \llbracket a, b \rrbracket=\{x \in \mathbb{Z}, x \in[a, b]\}$.

\section{At the Beginning...}

\section{A. Definitions}

Let $s$ be a discrete signal containing $N$ samples:

$$
s=\left[s_{1}, \ldots, s_{N}\right]^{T} .
$$

In the following, $s$ is of constant modulus. In other words, $s$ belongs to an hypertorus $\boldsymbol{T}^{N}$ of dimension $N$, which is a nonconvex set. Besides, each of its elements $s_{n}$ is only determined by a phase $\varphi_{n} \in[-\pi, \pi]$, i.e.:

$$
s_{n}= \begin{cases}1 / \sqrt{N} \exp \left(j \varphi_{n}\right) & \text { if } n \in \llbracket 1, N \rrbracket, \\ 0 & \text { otherwise. }\end{cases}
$$

Pulse compression is achieved by correlating the received signal with the transmitted pulse $s$. Basically, it consists in generating a sequence $\boldsymbol{y}$ of length $2 N-1$ such that:

$$
\boldsymbol{y}=\boldsymbol{\Lambda}_{N}(s) s^{*},
$$

where $\boldsymbol{\Lambda}_{N}(\boldsymbol{s})$ is a matrix of size $2 N-1 \times N$ containing delayed versions of sequence $s$, defined below.

$$
\boldsymbol{\Lambda}_{N}(s)=\left[\begin{array}{cccc}
s_{N} & 0 & \cdots & 0 \\
\vdots & \ddots & \ddots & \vdots \\
\vdots & & \ddots & 0 \\
s_{1} & \cdots & \cdots & s_{N} \\
0 & \ddots & & \vdots \\
\vdots & \ddots & \ddots & \vdots \\
0 & \cdots & 0 & s_{1}
\end{array}\right]
$$

In order to measure the sidelobe level of correlation sequence $\boldsymbol{y}$, the following criterion is considered, based on the $\ell_{p}$-norm, with $p \in \mathbb{N}^{*}$ :

$$
\begin{aligned}
\mathbb{C}^{N} & \rightarrow \mathbb{R}+ \\
\boldsymbol{s} & \mapsto f_{p}(\boldsymbol{s}):=\|\boldsymbol{F} \boldsymbol{y}\|_{p},
\end{aligned}
$$

where $\boldsymbol{F}$ is a diagonal matrix of order $2 N-1$, defined by the vector $[1, \ldots, 1,0,1, \ldots, 1]^{T}$, with all entries equal to 1 , except for a 0 at the entry $N$ (to discard the mainlobe). Notice that $f_{p}$ reduces to some known criteria for specific values of $p$, such as the Integrated Sidelobe Level (ISL) with $p=2$ or the Peak-to-Sidelobe-Level Ratio (PSLR) with $p=\infty$.

\section{B. Model}

As mentioned earlier, an eclipse is defined as a partial loss of data, due to an ongoing transmission. If it is at the beginning of the pulse, the returned signal may be written as:

$$
s_{\text {left }}^{i}=\left[0, \ldots, 0, s_{i+1}, \ldots, s_{N}\right]^{T} \in \mathbb{C}^{N} .
$$

Index $i$ counts the number of missing elements (or the number of zeros), compared to the complete sequence $s$. The ratio $i / N$ is known as the eclipsing ratio.

Remark: A discrete sequence describes a continuous signal in practice, with a certain sampling. For ease of simplicity, off-grid-eclipsing ratios are not considered in this paper, which might be overcome with an oversampling.

Reception processing is performed as if received signal $s_{\text {left }}^{i}$ is full. Its output is a sequence of length $2 N-1$, denoted $\boldsymbol{y}^{i}$, such that:

$$
\boldsymbol{y}^{i}:=\boldsymbol{\Lambda}_{N}(\boldsymbol{s})\left(\boldsymbol{s}_{\mathrm{left}}^{i}\right)^{*} .
$$

Notice that the previous expression links the energetic loss (due to the mismatch occurring between the received signal and the processing filter) and the eclipsing ratio, since:

$$
y_{N}^{i}=\sum_{n=i+1}^{N} s_{n} s_{n}^{*}=\frac{N-i}{N} .
$$

A definition of the correlation sidelobes with eclipses should depend on this normalisation, that is:

$$
\begin{aligned}
\mathbb{C}^{N} & \rightarrow \mathbb{R}+ \\
\boldsymbol{s} & \mapsto f_{p}^{i}(\boldsymbol{s}):=\left\|\frac{1}{y_{N}^{i}} \boldsymbol{F} \boldsymbol{y}^{i}\right\|_{p},
\end{aligned}
$$

so that the resulting level of sidelobes is maximised with respect to the corresponding mainlobe level. 


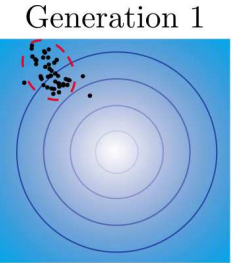

Generation 4

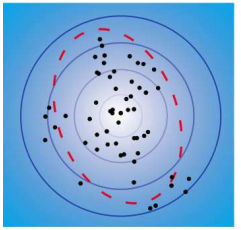

Small values of the cost function are represented in light colors.

In order to search for robust sequences to the eclipsing phenomenon, the following optimisation problem may be considered:

$$
\left\{\begin{aligned}
\min _{s} & f_{p}^{i}(s) \\
\text { t.q. } & s \in \boldsymbol{T}^{N}
\end{aligned}\right.
$$

that solves the sidelobe problem for one specific eclipsing ratio. However, like the delay parameter, the eclipsing ratio depends on the position of the target, which varies in practice. Optimising a sequence for only one ratio is a nonsense ! Several ratios should be considered simultaneously. Then, let $I$ be a set of indices $i$ ( $i$ refers to the number of missing elements). The problem becomes:

$$
\left\{\begin{aligned}
\min _{\boldsymbol{s}} & \left\{f_{p}^{i}(\boldsymbol{s})\right\}_{i \in I} \\
\text { t.q. } & \boldsymbol{s} \in \boldsymbol{T}^{N}
\end{aligned}\right.
$$

Expression (11) is a multi-objective optimisation problem [6]. As each cost function measures an identical quantity (a sidelobe level), it is possible to optimise the $\ell_{p}$-norm of all sidelobes, that is:

$$
\left\{\begin{array}{cl}
\min _{\boldsymbol{s}} & \left(\sum_{i \in I}\left(f_{p}^{i}(s)\right)^{p}\right)^{1 / p}, \\
\text { s.t. } & \boldsymbol{s} \in \boldsymbol{T}^{N} .
\end{array}\right.
$$

\section{Simulations Results}

In this subsection, several simulations have been performed to show the usefulness of the approach. Note first that the optimisation problem (12) in hand ...

- ... is non convex, because of the constant modulus constraint on sequence $s$. It means that it is quite difficult to determine the global minimum of the problem.

- ... has generally a lot of variables, depending on the length of $s$. Performance of optimisation methods may be deteriorated.

- ... is not separable. Separability is a property that allows to write a multivariate function as a product of monovariate functions. It thus helps solving large-scale optimisation problems [8].

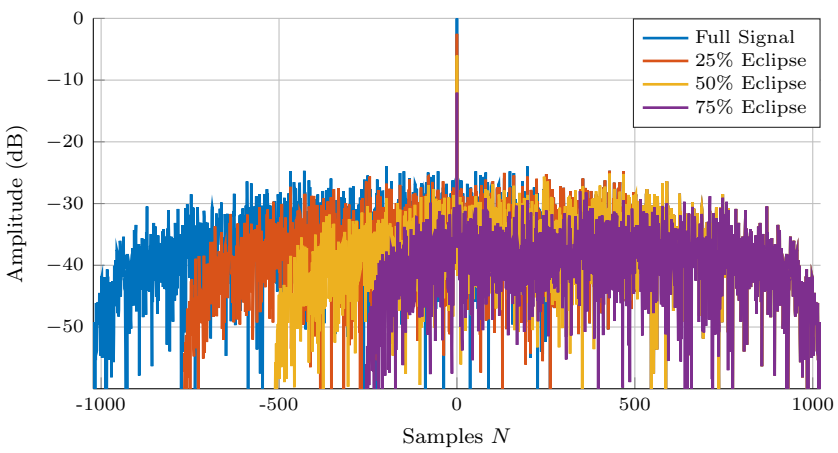

Random initialisation.

Correlation sequences are not normalised, to illustrate the energetic loss according to the eclipsing ratio.

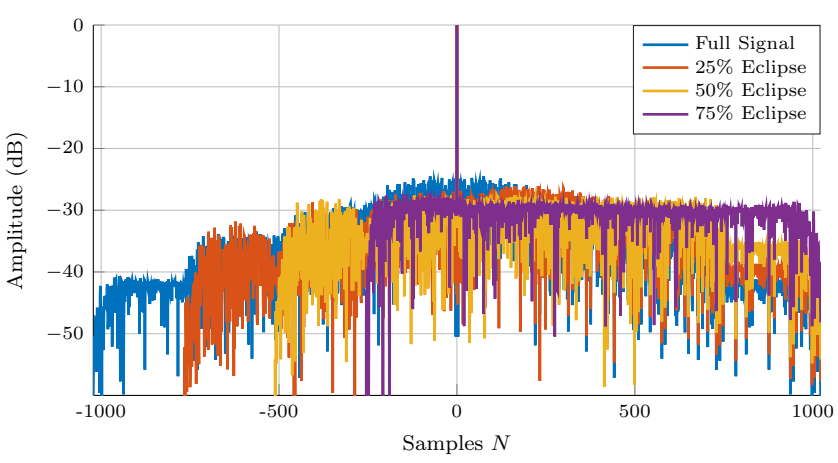

Obtained sequences.

Correlation sequences are this time normalised.

Figure 4. Optimisation of a sequence with several eclipsing ratios

In a nutshell, none of the optimisation methods can guarantee a global convergence in practice, to the best of our knowledge. In the following, an evolutionary algorithm named CMA-ES has been applied [9] (CMA-ES stands for Covariance Matrix Adaptation Evolution Strategy). As a randomised black box search algorithm, it is quite convenient since it only needs the outputs of the cost function. Fig. 3 illustrates some principles of the CMA-ES algorithm.

- At start, a random population is generated, according to a multivariate normal distribution (represented in the figure via ellipses).

- Selection: As an evolution strategy, best candidates (in the sense of the cost function) are selected. They update some parameters of the search distribution (such as the mean or the covariance matrix).

- Generation: From this distribution, a new population is generated.

- This process is repeated until convergence.

In the following example, an optimisation of a sequence $s$ of length $N=1023$ is performed, with respect to four eclipsing ratios: $0 \%$ (which is equivalent to the full sequence), $25 \%$, $50 \%$ and $75 \%$. In Fig. 4, correlation sequences are presented, whilst Fig. 5 plots the PSLR according to several eclipsing ratio. As concerns the random initialisation, the sidelobe level goes on the same way as the eclipsing ratio: if one increases, 


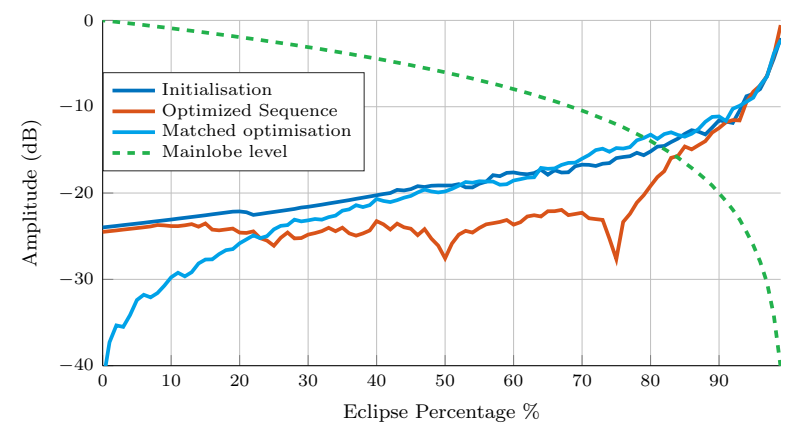

Figure 5. Sidelobe level with several eclipsing ratios Robustness of an optimised sequence in the matched filter sense [7] is also depicted.

the other does too. As concerns the optimised sequence, the sidelobe level seems to be more consistent, except for the highest ratios that were not considered in the constraints (the energetic loss becomes too substantial). Besides, an improvement between 4 and $11 \mathrm{~dB}$ can be observed, compared to the initialisation. Notice that the peak sidelobe of the full sequence is around $-25 \mathrm{~dB}$, whilst it may be measured at $-43 \mathrm{~dB}$ if only one ratio is considered. Joys of compromise...

\section{III. ... AND AT THE END}

\section{A. Model Extension}

An eclipse is defined as an incomplete reception. Section II describes only the particular case where the beginning of the received signal is missing. But a loss may also be set at its end, as depicted in Fig. 6.

In this regard, it is preferable that the transmitted signal is robust to both types of eclipses. Thus, an extension of the previous procedure is suggested in this section. The received signal is of the following model:

$$
\begin{array}{ll}
\boldsymbol{s}_{\text {left }}^{i}=\left[0, \ldots, 0, s_{i+1}, \ldots, s_{N}\right]^{T} \in \mathbb{C}^{N}, & \text { (at the begin.) } \\
\boldsymbol{s}_{\text {right }}^{j}=\left[s_{1}, \ldots, s_{N-j}, 0, \ldots, 0\right]^{T} \in \mathbb{C}^{N}, & \text { (at the end) }
\end{array}
$$

where integers $i$ and $j$ are derived from eclipsing ratios. Let $J$ be a set of indices $j$, and define $\left\{g_{p}^{j}\right\}_{j \in J}$, a set of objective functions that are associated to $s_{\text {rig. }}^{j}$.

Once again optimising the norm of all sidelobes, the optimisation problem becomes:

$$
\left\{\min _{s} \sum_{i \in I}\left(f_{p}^{i}(s)\right)^{p}+\sum_{j \in J}\left(g_{p}^{j}(s)\right)^{p}\right)^{1 / p},
$$

\section{B. Simulations Results}

In this paragraph, a short comparison of the two methods is proposed. Optimisation is applied on a sequence of length $N=127$, with the four same eclipsing ratios, i.e. $0 \%, 25 \%$, $50 \%$ and $75 \%$. Figs. 7-8 show some processing outputs of sequences that are optimised for eclipses at the beginning and both, respectively.

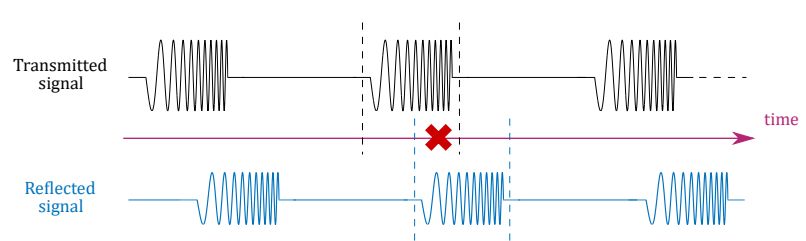

An eclipse at the beginning of the received pulse

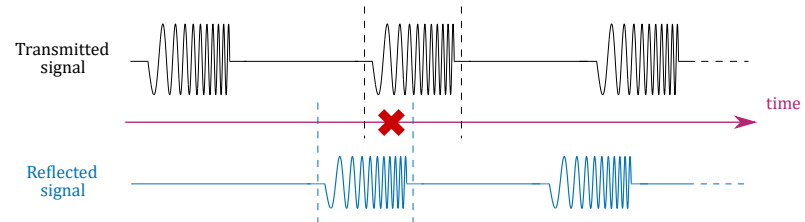

An eclipse at the end of the received pulse

Figure 6. Different types of eclipses

As concerns the eclipse at the beginning, the latter procedure is behind, with a difference of $3 \mathrm{~dB}$. As concerns the eclipse at the end, the trend is obviously reversed. Whilst sidelobes are quite preserved in the second model $(-16 \mathrm{~dB}$, in the PSLR sense), they are totally uncontrolled in the other $(-5 \mathrm{~dB}$ in this example, which is equivalent to a random sequence). There results are not-so-surprising, since these sidelobes are not accounted for in the cost function of (12).

\section{Conclusion}

In this paper, a method has been proposed in order to generate polyphase codes that are quite robust to the eclipsing phenomenon. It is based on an optimisation problem, that should consider eclipses at both the beginning and the end of the pulse. Simulations show some improvements in the context of eclipses. But this robustness implies a noticeable decrease of detection performance in the ideal case.

Future works include two extensions of the suggested procedure, which still aim to limit the effects of eclipsing:

- Optimisation of a mismatched filter, on top of considering the transmitted sequence;

- Optimisation of a set of phase codes for a coherent MIMO radar.

\section{ACKNOWLEDGEMENT}

This work was funded by Thales Land \& Air Systems and was partly supported by a DGA-MRIS scholarship.

\section{REFERENCES}

[1] Merriam-Webster, "Eclipse," online, Dec. 2018, https://www.merriamwebster.com/dictionary/eclipsing.

[2] M. Skolnik, Introduction to Radar Systems, ser. Electrical Engineering Series. McGraw-Hill, 2001.

[3] S. D. Blunt, K. Gerlach, and E. L. Mokole, "Pulse Compression EclipsingRepair," in 2008 IEEE Radar Conference, May 2008, pp. 1-5.

[4] C. M. Alabaster and E. J. Hughes, "Generating Pulse Compression Waveforms Robust to Eclipsing," in International Conference on Radar Systems (Radar 2017), Oct 2017, pp. 1-6.

[5] R. K. Shokooh and M. Okhovvat, "Modified-Adaptive Pulse Compression Repair Algorithm Based on Post-Processing for Eclipsing Effects," IET Radar, Sonar Navigation, vol. 12, no. 12, pp. 1527-1534, 2018. 

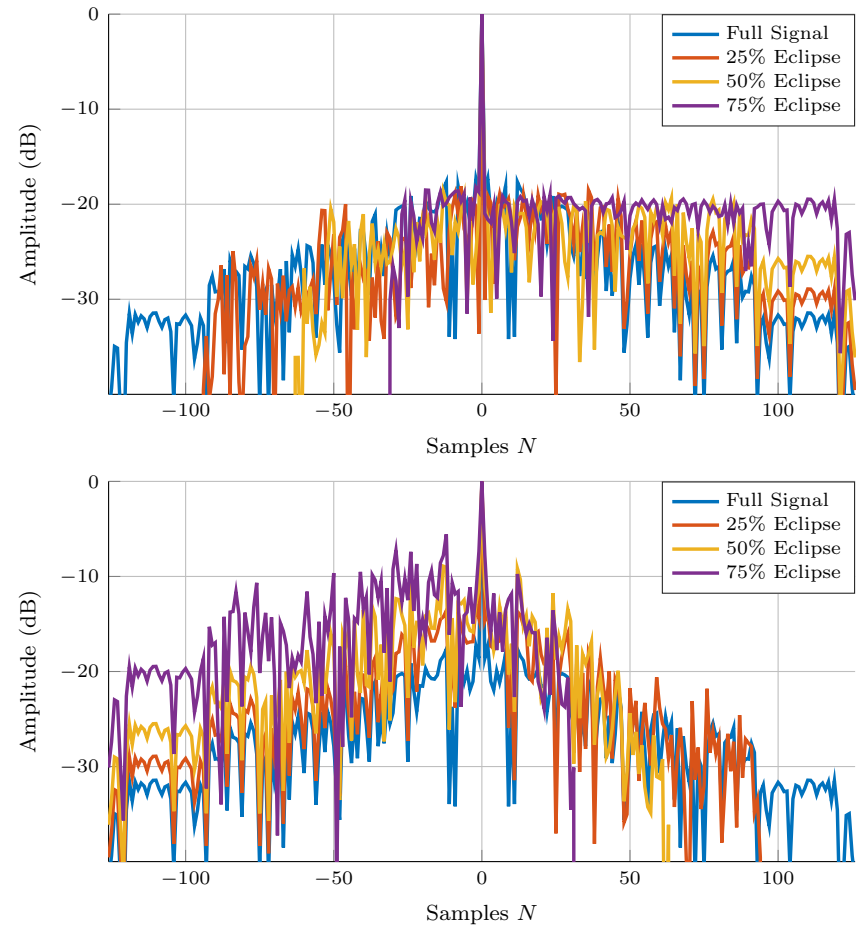

Figure 7. Optimisation for an eclipse at the beginning only On the top: correlation sequences for an eclipse at the beginning of the received pulse. On the bottom: correlation sequences for an eclipse at the end of the received pulse.
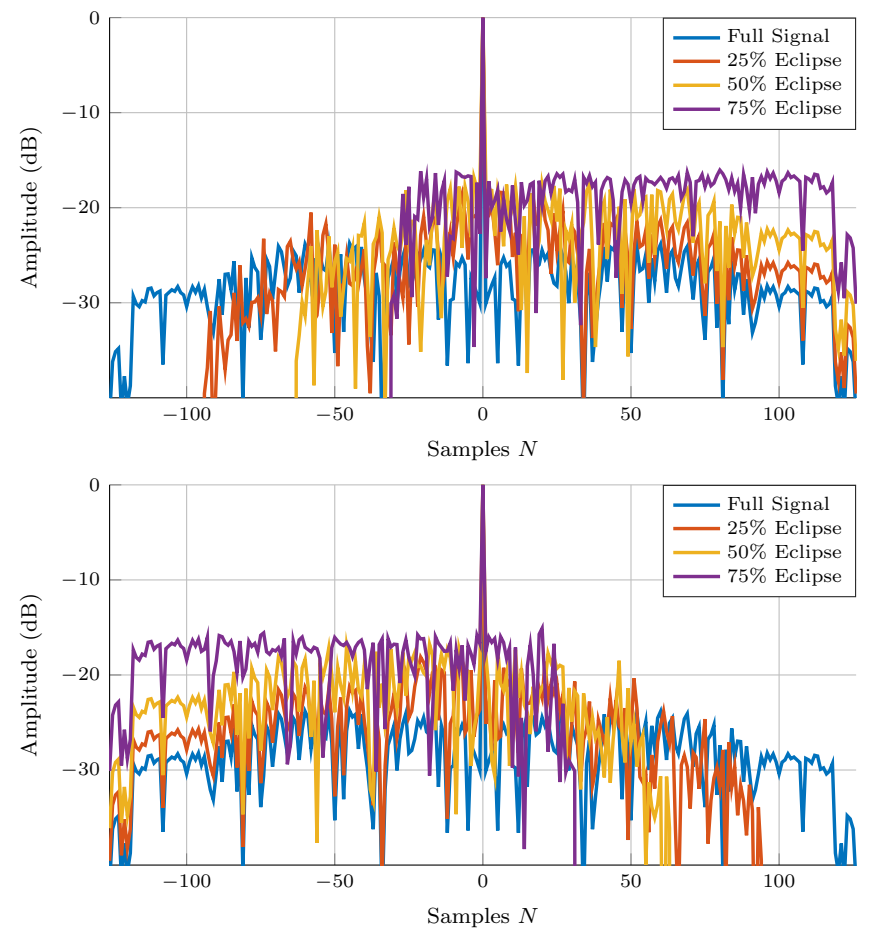

Figure 8. Optimisation for an eclipse at both the beginning and the end On the top: correlation sequences for an eclipse at the beginning of the received pulse. On the bottom: correlation sequences for an eclipse at the end of the received pulse.
[6] Y. Collette and P. Siarry, Multiobjective Optimization: Principles and Case Studies, ser. Decision Engineering. Springer Berlin Heidelberg, 2004.

[7] J. M. Baden, M. S. Davis, and L. Schmieder, "Efficient Energy Gradient Calculations for Binary and Polyphase Sequences," in Radar Conference (RadarCon), 2015 IEEE, May 2015, pp. 0304-0309.

[8] V. Tsurkov, Large-scale Optimization: Problems and Methods, ser. Applied Optimization. Springer US, 2013.

[9] N. Hansen, "The CMA Evolution Strategy: A Tutorial," CoRR, vol. abs/1604.00772, 2016. [Online]. Available: http://arxiv.org/abs/1604. 00772 